\title{
Secondary central nervous system lymphoma surrounding a region injured by subarachnoid hemorrhage and subsequent aneurysmal clipping
}

\author{
YORIKO MURATA ${ }^{1}$, YASUHIRO HATA ${ }^{2}$, YOSHIHIRO NODA ${ }^{2}$, SATOSHI MATSUSAKA $^{2}$, NAOKI FUKUI ${ }^{3}$, \\ TOMOHITO KADOTA $^{3}$, JUN IWATA ${ }^{4}$, TAKUYA MACHIDA ${ }^{5}$ and TAKUJI YAMAGAMI ${ }^{1}$
}

${ }^{1}$ Department of Radiology, Kochi Medical School, Kochi 7838505; ${ }^{2}$ Department of Radiology, Kochi Health Sciences Center,
Kochi 7818555; ${ }^{3}$ Department of Neurosurgery, Kochi Medical School, Kochi 7838505; ${ }^{4}$ Department of Pathology;
${ }^{5}$ Department of Hematology and Transfusion Medicine, Kochi Health Sciences Center, Kochi 7818555, Japan

Received August 18,2017; Accepted August 30, 2017

DOI: 10.3892/br.2017.981

\begin{abstract}
The present case study describes a rare case of secondary central nervous system (CNS) lymphoma that infiltrated the dura and leptomeninges around the area injured by subarachnoid hemorrhage and subsequent aneurysmal clipping. Invasion of the CNS was observed by computed tomography as slurred fissures of the right parietal lobe adjacent to the surgery area. Subdural and subarachnoid enhancement overlapping the area injured by past surgical procedures was observed by contrast-enhanced magnetic resonance imaging. Surgical resection revealed B-cell lymphoma infiltrating the dura and leptomeninges surrounding the post-hemorrhagic area. The patient was subsequently diagnosed with systemic lymphoma and bone marrow invasion, and multiple lymph node swelling. To the best of our knowledge, this is the first report of malignant lymphoma involving the CNS overlapping a previously injured area.
\end{abstract}

\section{Introduction}

Leptomeningeal infiltration is frequently encountered (1), and dural infiltration is not rare with secondary central nervous system (CNS) lymphoma $(1,2)$. However, unless systemic lymphoma has already been identified, a finding of subdural and subarachnoid abnormality would not be directly linked to lymphoma (3). Furthermore, computed tomography (CT) and magnetic resonance imaging (MRI) abnormalities surrounding hemorrhagic and postoperative changes make it difficult to distinguish between onset of disease and postoperative complications, including metal artifacts. However, malignant

Correspondence to: Dr Yoriko Murata, Department of Radiology, Kochi Medical School, 185-1 Kohasu, Kochi 7838505, Japan

E-mail: muratay@kochi-u.ac.jp

Key words: malignant lymphoma, central nervous system invasion, history of subarachnoid hemorrhage, disrupted blood brain barrier cells that enter the CNS appear first in the dura and subarachnoid space in rats with a blood brain barrier (BBB) disrupted by focal injury following exposure to a cold temperature (4). Previous history of subarachnoid hemorrhage (SAH) and aneurysm clipping may be associated with CNS infiltration in systemic lymphoma. The current study describes a particularly rare case of systemic lymphoma involving the CNS overlapping with the area of injury to the dura and leptomeninges due to an aneurysmal SAH. To the best of our knowledge, no similar cases have previously been reported.

\section{Case report}

A 56-year-old woman with a history of aneurysm clipping following acute $\mathrm{SAH}$ due to rupture of the right middle cerebral arterial aneurysm 6 years earlier (Fig. 1) was hospitalized at Kochi Health Sciences Center for sudden numbness of the left arm. The patient had not experienced malignant or benign diseases within the 6 years since discharge from hospital. The patient had been asymptomatic except for partial paralysis due to SAH. Unenhanced CT was performed to exclude the recurrence of $\mathrm{SAH}$, and it revealed slurred fissures of the right parietal region and enlargement of the low-density area surrounding the preceding hemorrhagic scar was suspected (Fig. 2). In addition, MRI was performed (Fig. 3). Diffusion-weighted imaging (DWI) demonstrated a thin crescent of hyperintensity in the right temporo-occipital region, although the parenchyma near the right Sylvian fissure exhibited loss of signal with distortion due to a magnetic susceptibility artifact. Fluid-attenuated inversion recovery (FLAIR) demonstrated slurred fissures of the right temporo-occipital region, and hyperintensity near the Sylvian fissure. T2-weighted gradient echo imaging (T2WI) demonstrated linear hypointensities along the surface of the right cerebral hemisphere as superficial siderosis from hemosiderin deposits caused by SAH. Enhanced axial T1-weighted imaging demonstrated intense homogeneous enhancement along the dura, and subarachnoid linear enhancement in the right temporo-occipital region. A subdural mass showing enhancement was strongly suspected. However, 

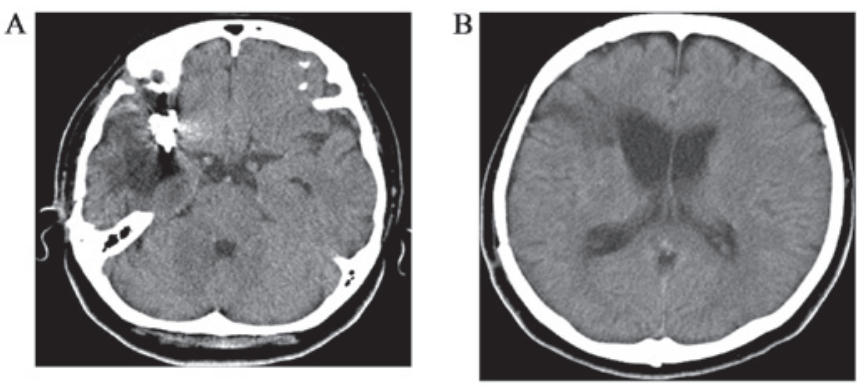

Figure 1. Brain computed tomography 4 months after acute subarachnoid hemorrhage and aneurysm clipping. (A) Intense hyperdensity from aneurysm clipping was apparent in the right frontotemporal region. (B) A hypodense area reflecting gliosis from acute hemorrhage revealed adjacent to clipping part.
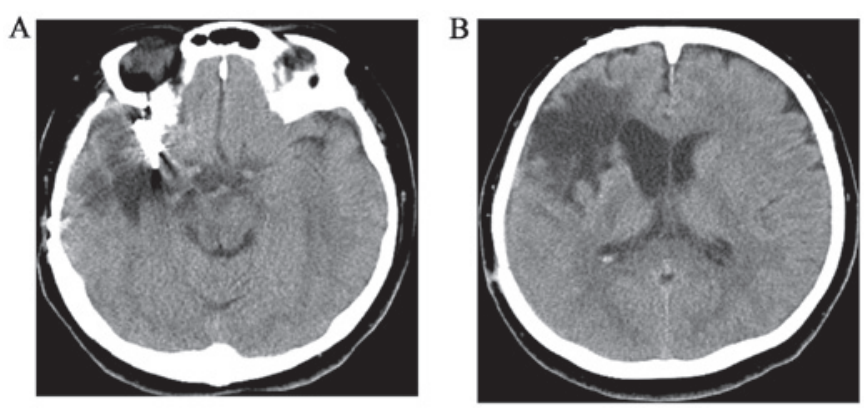

Figure 2. Brain CT obtained 6 years after that demonstrated in Fig. 1. (A) CT revealed aneurysm clipping in right frontotemporal region like Fig. 1. (B) Enlarged hypodense area revealed near the Sylvian fissure. CT, computed tomography.
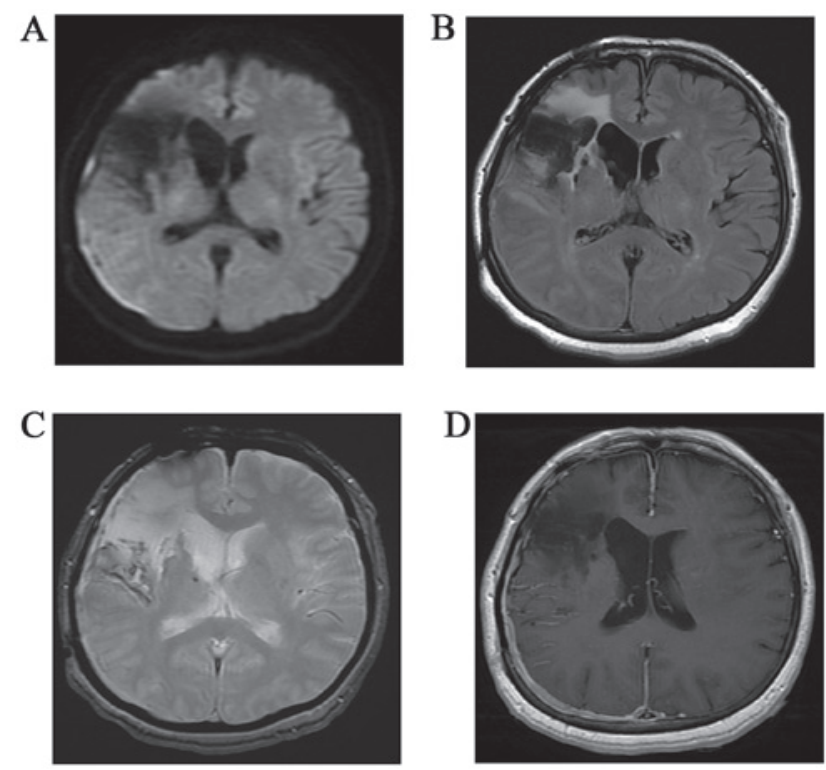

Figure 3. Brain magnetic resonance imaging obtained following the brain computed tomography scan obtained in Fig. 2. (A) The thin crescent of hyperintensity revealed in the right temporo-occipital region and the parenchyma near the right Sylvian fissure represent loss of signal with distortion by magnetic susceptibility artifact on diffusion-weighted imaging. (B) Fluid-attenuated inversion recovery demonstrated slurred fissures in the right temporo-occipital region and hyperintensity near the Sylvian fissure. (C) Linear hypointense signals were revealed along the surface of the right cerebral hemisphere as superficial siderosis from hemosiderin deposits by SAH upon T2-weighted gradient echo imaging (WI). (D) Intense homogeneous enhancement along the dura were apparent, along with subarachnoid linear enhancements in the right temporo-occipital region upon enhanced T1WI. subarachnoid enhancement or FLAIR hyperintensity near the right Sylvian fissure resulted in confusion between the presence of novel lesions or postoperative complications. Prior to surgery, meningioma with postoperative change was suspected on the basis of subdural hyperintensity on DWI. Intraoperatively, the dura mater and subarachnoid space exhibited a grayish-yellow mass, and malignant lymphoma was diagnosed pathologically. Subsequent systemic examination by gallium scintigraphy and CT revealed multiple mediastinal and paraaortic lymph node swellings. Following CT, neck lymph nodes began to demonstrate rapid increases in size. Serum interleukin-2 receptor and $\beta 2$-microglobulin levels were high, at 2,170 mg/l (normal, $<519 \mathrm{mg} / \mathrm{ml}$ ) and $5.0 \mathrm{U} / \mathrm{ml}$ (normal, <1.9 U/ml), respectively. Bone marrow findings were consistent with CNS lymphoma (non-Hodgkin's B-cell type; stage IV). Based upon positive results for cyclin D1 postoperatively, mantle cell lymphoma was proposed, although the subtype was not confirmed. At the time of writing, the patient is undergoing systemic chemotherapy with a combination of rituximab, methotrexate and cytalabine.

\section{Discussion}

Subdural and subarachnoid enhancement on MRI may be indicative of a variety of differential diagnoses from benign to malignant, such as sarcoidosis, meningitis and metastatic tumors with leptomeningeal dissemination other than secondary lymphoma (5). MRI findings were consistent with CNS invasion by lymphoma (2); however, lymphoma were not considered amongst the more likely options. CT or MRI abnormalities overlapping a previously injured region indicating a complication from surgery could not be excluded. In the present case, subdural crescent enhancement identified on MRI were exhibited as slurred fissures by CT. In addition, DWI was distorted by magnetic susceptibility artifacts; in retrospect, the subarachnoid findings of FLAIR and the enhanced MRI were consistent with the surgical features of leptomeningeal infiltration (6). Prominent subarachnoid enhancement on contrast-enhanced T1WI may depict evidence of malignancy; however, her past history had to be taken into account.

Should systemic lymphoma be revealed in advance, invasion of the CNS may be included in the differential diagnosis, regardless of how distorted the CT or MRI findings are. Although primary leptomeningeal or dural lymphoma is rare $(7,8)$, secondary CNS invasion is not. CNS involvement in non-Hodgkin's lymphoma tends to occur early, at a median of 5-6 months subsequent to the primary diagnosis of systemic lymphoma (3). Lymphoma in the current patient would represent an aggressive subtype according to the rapid increase in the size of neck lymph nodes.

Exactly why secondary lymphoma tends to present the dural or leptomeningeal spread remains unknown. However, lymphoma cells hypothetically spread from retroperitoneal lymph nodes or bone marrow to the leptomeninges via the intervertebral nervous plexus (9). Aho et al (4) reported that malignant cells appeared to enter the CNS through a deficiency in the BBB around the subarachnoid vessels. Composite meningioma and lymphoma has been reported as a form of tumor-to-tumor metastasis (10). Previous disruption of the 
BBB by meningioma may be considered as the grounds for invasion of CNS lymphoma. In the present case, the BBB of the broad area surrounding the right Sylvian fissure had been disrupted by acute SAH and subsequent surgery 6 years earlier. CNS invasion by lymphoma may be associated with disruption of the BBB. Although confirmatory evidence is lacking, the possibility remains that subdural and leptomeningeal invasion of lymphoma occurred through disruption of the BBB by SAH and subsequent clipping.

In conclusion, the current study presents a case of CNS invasion by systemic lymphoma that was difficult to radiologically diagnose due to overlap with an area previously affected by SAH and a subsequent surgical scar. It is proposed that radiologists assess the possibility of invasion by CNS lymphoma in the presence of meningeal abnormality overlapping an injured region.

\section{References}

1. Haldorsen IS, Espeland A and Larsson EM: Central nervous system lymphoma: Characteristic findings on traditional and advanced imaging. AJNR Am J Neuroradiol 32: 984-992, 2011.

2. Koeller KK, Smirniotopoulos JG and Jones RV: Primary central nervous system lymphoma: Radiologic-pathologic correlation. Radiographics 17: 1497-1526, 1997.
3. Hill QA and Owen RG: CNS prophylaxis in lymphoma: Who to target and what therapy to use. Blood Rev 20: 319-332, 2006.

4. Aho R, Vaittinen S, Jahnukainen K and Kalimo H: Spread of malignant lymphoid cells into rat central nervous system with intact and disrupted blood-brain barrier. Neuropathol Appl Neurobiol 20: 551-561, 1994.

5. Guermazi A, Lafitte F, Miaux Y, Adem C, Bonneville JF and Chiras J: The dural tail sign--beyond meningioma. Clin Radiol 60: 171-188, 2005.

6. Stuckey SL, Goh TD, Heffernan T and Rowan D: Hyperintensity in the subarachnoid space on FLAIR MRI. AJR Am J Roentgenol 189: 913-921,2007.

7. Taylor JW, Flanagan EP, O'Neill BP, Siegal T, Omuro A Deangelis L, Baehring $J$, Nishikawa $R$, Pinto $F$, Chamberlain M, et al: Primary leptomeningeal lymphoma: International Primary CNS Lymphoma Collaborative Group report. Neurology 81: 1690-1696, 2013.

8. Johnson BA, Fram EK, Johnson PC and Jacobowitz R: The variable MR appearance of primary lymphoma of the central nervous system: Comparison with histopathologic features. AJNR Am J Neuroradiol 18: 563-572, 1997.

9. Levitt LJ, Dawson DM, Rosenthal DS and Moloney WC: CNS involvement in the non-Hodgkin's lymphomas. Cancer 45: $545-552,1980$.

10. Martin SE, Khalidi HS and Hattab EM: Marginal zone B-cell lymphoma involving a longstanding fibrous meningioma: An initial manifestation of systemic disease. Hum Pathol 44: 2609-2613, 2013 